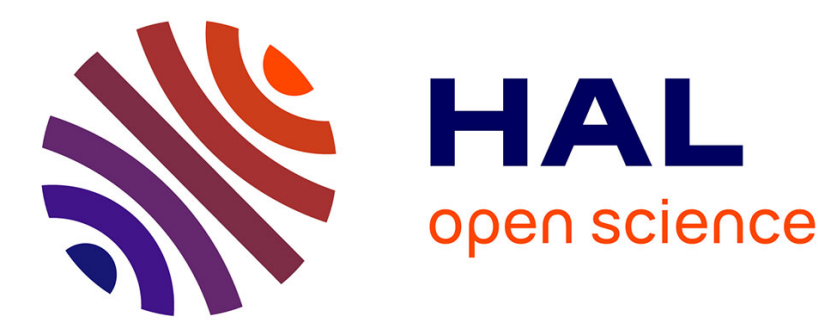

\title{
Le spectre des croyances religieuses
}

Paul Clavier

\section{To cite this version:}

Paul Clavier. Le spectre des croyances religieuses. Philosophie, 2020, Nº145 (2), pp.91-101. 10.3917/philo.145.0091 . hal-02977408

\section{HAL Id: hal-02977408 \\ https://hal.science/hal-02977408}

Submitted on 29 Oct 2020

HAL is a multi-disciplinary open access archive for the deposit and dissemination of scientific research documents, whether they are published or not. The documents may come from teaching and research institutions in France or abroad, or from public or private research centers.
L'archive ouverte pluridisciplinaire HAL, est destinée au dépôt et à la diffusion de documents scientifiques de niveau recherche, publiés ou non, émanant des établissements d'enseignement et de recherche français ou étrangers, des laboratoires publics ou privés. 
Paul Clavier

Le spectre des croyances religieuses

Les croyances religieuses font l'objet de nombreuses considérations de la part de philosophes, d'anthropologues, de sociologues, de psychologues, de neuroscientifiques... On ne leur épargne aucun traitement et on peut parler à cet égard d'un véritable acharnement théorique : traitement propositionnel, traitement existentiel, traitement analytique, traitement herméneutique, traitement métaphysique, traitement anti-métaphysique, recherche des conditions de vérité, quête de sens, logique des prédicats, formes de vie, explication par les conditionnements socio-linguistiques ou neurophysiologiques, compréhension par les choix de valeurs...

Je ne pense pas qu'on puisse dire a priori quel traitement est le plus approprié. Et il me paraît sage de garder à l'esprit qu'il n'y a pas un unique type de croyance religieuse. L'objet de cette intervention est de rappeler la grande diversité du spectre des croyances religieuses. Et de militer pour un particularisme méthodologique : en clair de recommander l'étude de cas, au détriment des séduisantes gigantomachies opposant ou réconciliant Foi et Savoir, Croyance et Rationalité. La grande oubliée de la discussion sur la croyance religieuse, c'est la personne croyante. Une même croyance, par exemple la croyance en un créateur, pourra relever chez l'une d'une réflexion métaphysique, chez l'autre d'un besoin de sécurité, chez l'autre encore d'une conception éthique ou écologique, d'un sentiment de dépendance absolue (Schleiermacher), pourquoi pas d'une stratégie adaptative neuronale, ou d'un suivisme sociétal. Il y a des cas où l'analyse logique et définitionnelle est très éclairante (comme dans le cas du concept de toute-puissance). D'autres où l'exégèse scripturale, la théologie spirituelle, l'ethnolinguistique, la sociologie seront plus pertinentes.

S'il est téméraire de prétendre proposer une conceptualisation complète des croyances religieuses, il peut être opportun d'en suggérer une possible représentation d'ensemble du spectre des croyances, en revisitant plusieurs distinctions ou polarités.

1. Noyau propositionnel public ou bénéfice subjectif individuel ? Commençons par la distinction canonique entre les croyances qui seraient ouvertes à la discussion publique rationnelle et celles qui, par nature réfractaire à un examen en termes de critères d'acceptabilité rationnelle, relèveraient d'un bénéfice psychologique subjectif, que Max Weber appelait les récompenses psychologiques (psychologische Prämien). Je propose d'illustrer cette distinction au moyen de deux paraboles empruntées à deux « philosophes de la religion » distants de deux siècles : l'apologue du Souper de Bassora (extrait du Zadig de Voltaire, 1747) et celui du meeting interreligieux dû à Simon Blackburn. 
Un souper donné dans la ville de Bassora réunit des négociants de toutes contrées et de toutes religions : un Égyptien, un Indien du Gange, un habitant du Cathay, un Grec, un Celte, et plusieurs autres étrangers. L'Egyptien, très remonté par ce qu'on a refusé de lui prendre en gage la momie de sa défunte tante, s'apprête à manger une poule. L'Indien veut l'en empêcher car il se pourrait que l'âme de la défunte fût passée dans le corps de cette poule. L'Egyptien rétorque que, dans sa religion, l'adoration du boeuf Apis n'interdit pas de manger un bon steack. L'Indien invoque l'antiquité de Brama. Le Chaldéen lui oppose alors Oannès, poisson divin à tête d'homme et à queue dorée, qu'il faut adorer, pourvu qu'on ne fasse jamais cuire de poisson. Le Celte, lui, veut imposer le gui des cultes druidiques. L'homme de Cambalu, adepte du Li et du Tien, sous entend que sa religion n'est pas moins ancienne que celle des autres, mais que le bonheur est plus vénérable encore que la plus haute antiquité. Le Grec assure alors péremptoirement que "le chaos est le père de tout, et que la forme et la matière ont mis le monde dans l'état où il est », ce qui, après tout, n'est pas une mauvaise description de la situation.

Zadig intervient donc pour calmer le jeu et va démêler l'écheveau de ces croyances religieuses rivales : "Mes amis, vous alliez vous quereller pour rien, car vous êtes tous du même avis. "À ce mot, ils se récrièrent tous. "N'est-il pas vrai, dit-il au Celte, que vous n'adorez pas ce gui, mais celui qui a fait le gui et le chêne? - Assurément, répondit le Celte. - Et vous, monsieur l'Égyptien, vous révérez apparemment dans un certain bœuf celui qui vous a donné les boufs? - Oui, dit l'Égyptien. - Le poisson Oannès, continua-t-il, doit céder à celui qui a fait la mer et les poissons. - D'accord, dit le Chaldéen. - L'Indien, ajouta-t-il, et le Cathayen, reconnaissent comme vous un premier principe; je n'ai pas trop bien compris les choses admirables que le Grec a dites, mais je suis sûr qu'il admet aussi un Être supérieur, de qui la forme et la matière dépendent. " Le Grec, qu'on admirait, dit que Zadig avait très-bien pris sa pensée. "Vous êtes donc tous de même avis, répliqua Zadig, et il n'y a pas là de quoi se quereller. " L'intervention de Zadig vise ici un noyau propositionnel commun: les religions seraient vraies en ce qu'elles affirment, et fausses en ce qu'elles nient. On peut bien sûr se demander si cette entreprise de réduction à un dénominateur commun vide la croyance religieuse de sa singularité historique, culturelle et du vécu individuel ? Nous avons ici en tout cas le projet d'un syncrétisme par consultation, puisque Zadig recueille un consensus, et prétend mettre à jour une croyance commune dispositionnelle, que les croyants sont prêts à reconnaître plus fondamentale que l'habillage rituel ou confessionnel dont cette croyance était jusqu'alors revêtue.

Venons-en à l'autre option qui, récusant cette possibilité, présente les croyances religieuses sous le seul aspect du bénéfice psychologique : «Les représentants des principales religions du monde sont réunis dans un débat 
télévisé. Le bouddhiste prend la parole et vante les voies de la maîtrise, du détachement et de l'extinction du soi. Les autres intervenants s'exclament en choeur: «Waoh fantastique! si ça marche pour toi, c'est géant ! (Wow, terrific, if that works for you that's great!) ». L'hindou parle alors de la métempsycose et des enseignements de Krishna. Les autres intervenants s'exclament en chœur : «Waoh fantastique! si ça marche pour toi, c'est géant !» Le catholique prend la parole et expose le message du Christ et la promesse du salut et de la vie éternelle. Les autres s'exclament en chœur : «Waoh fantastique! si ça marche pour toi, c'est géant». Mais le catholique tape alors du poing sur la table et s'écrie : «Mais non, la question n'est pas que cela marche pour moi ! C'est la vérité du Dieu vivant, et si vous n'y croyez pas vous serez damnés! ». Mais les autres intervenants reprennent: «Waoh fantastique! si ça marche pour toi, c'est géant ${ }^{1}$

La croyance religieuse est alors confinée exclusivement dans la sphère privée, et toute prétention à être une proposition universelle lui est refusée ${ }^{2}$. Mieux encore: le prosélytisme lui-même est considéré comme une conviction à usage strictement interne. Le refus de laisser sa croyance réduire à un bénéfice psychologique individuel ( si ça marche pour toi, c'est géant! ») est lui-même réduit à un bénéfice psychologique individuel. Le croyant (ici le catholique) prétend à la validité objective et universelle de ses croyances, ses auditeurs reconduisent cette prétention à une performance subjective. Le spectre des croyances religieuses est bien large, puisqu'il s'étend de l'objectivisme au subjectivisme et englobe aussi bien des propositions à validité publique que des énoncés à usage privé.

2. Perspective sur les croyances religieuses en première et en troisième personnes.

Bien entendu, ces deux options sont des cas-limites, même si certaines pratiques religieuses (le bahaïsme par exemple) ou théoriques (les entreprises de naturalisation de la croyance, le neuro-théologie etc.) semblent illustrer les deux extrémités du spectre (objectivisme universaliste vs accès privé à un bénéfice psychologique).

Mais cette antithèse est trop rigide. Il est juste de l'estomper. D'abord en revenant sur la notion de croyance privée. Ce n'est pas parce qu'une

\footnotetext{
${ }^{1}$ Simon Blackburn, Being Good : A Short Introduction To Ethics, Oxford University Press 2001, ch. I : "The Death of God", p. 26.

${ }^{2}$ Rudolf Bultmann, par exemple, entend vider la foi dans la création de son contenu métaphysique : «seuls sont légitimes les énoncés sur Dieu qui expriment la relation existentielle entre l'homme et Dieu. Illégitimes sont les énoncés qui parlent de l'action de Dieu comme d'un événement cosmique. L'affirmation de Dieu comme créateur ne peut pas être entendue comme un énoncé théorique sur Dieu, pris au sens général de creator mundi, mais elle exprime exclusivement une relation existentielle. Cette affirmation ne peut être qu'une confession personnelle signifiant que je me comprends moi-même comme une créature qui doit son existence à Dieu » (Jesus (1926), trad. fr. Editions du Seuil, Paris 1968, p. 232).
} 
croyance a le caractère d'une expérience privée qu'il faut lui refuser tout contenu propositionnel, et qu'elle est nécessairement indicible ou incommensurable. Chacune de nos perceptions auditives, visuelles, tactiles, ou mémorielles, ou émotionnelles, est privée : ce qui ne nous empêche pas de les comparer à celles d'autrui, et de nous demander si ce sont les perceptions (partielles) d'un monde commun, en formulant des propositions décrivant ce que nous percevons.

La personne croyante est, sauf simulation, le témoin irrécusable de ce qu'elle croit, de ce qu'elle se trouve croire, ou prête à croire. Mais l'accès en première personne ne préjuge pas de la nature de ce à quoi elle accède. Il peut y avoir un accès exclusivement privé à des contenus propositionnels, comme il pourrait $\mathrm{y}$ avoir un accès public à quelque chose de nonpropositionnel (le « sentiment océanique de l'existence» par lequel Freud caractérise la croyance religieuse). Il importe donc distinguer la perspective sur les croyances religieuses (en première personne ou en troisième personne) et le contenu de cette croyance (factuel ou expressif, existentiel ou propositionnel, descriptif ou prescriptif, etc.). Ce n'est pas parce qu'une croyance est privée qu'elle est non-propositionnelle. Ce n'est pas parce qu'elle est propositionnelle qu'elle est publique. On ne peut donc privatiser à volonté ni déprivatiser par décret les croyances religieuses. Il faut examiner.

Chesterton s'est fait le champion de la déprivatisation des croyances religieuses. Il déclare : «On ne construit pas une conception métaphysique du monde pour qu'elle aille à un individu, mais pour qu'elle colle avec le monde; il n'est pas plus au pouvoir d'un individu de se doter d'une religion privée que d'avoir son Soleil et sa Lune privée $»^{3}$.

Outre son engagement objectiviste fort, cette déclaration repose sur une prémisse implicite : toute religion contient une conception métaphysique du monde (Chesterton présuppose également : toute conception métaphysique du monde construite pour aller à un individu est privée).

Ce qui pose au moins deux problèmes. Premier problème : l'objectivisme métaphysique public de la religion selon Chesterton convient-il à toute religion? En la matière, il y a peut-être des religions plus métaphysiques que d'autre. Prenons un exemple. Certaines déclarations authentifient comme bouddhiste toute religion qui admet les quatre sceaux du Dharma, à savoir :1. Tous les phénomènes composés sont impermanents. 2. Tout ce qui est corrompu (c.-à-d. conditionné) est souffrance. 3. Tous les phénomènes sont dépourvus de soi. 4. Le nirvâna est paix. Mais sur la base de cet exemple, on ne va pas conclure que toute attitude religieuse est justiciable d'un noyau

${ }^{3}$ Gilbert Keith Chesterton, Introduction to the Book of Job (1916), réimprimé dans The Chesterton Review 11 (1985): 5-15. 
propositionnel. Une hirondelle ne fait pas le printemps. Car on ne saurait déterminer ce que veut dire « admetttre les quatre sceaux du darma ». Est-ce les réciter, les proclamer, les afficher à l'entrée des lieux de culte, les vivre, les enseigner?

Deuxième problème: à supposer même l'existence d'un noyau propositionnel qui serait définitoire d'une religion, peut-on l'isoler de tout le vécu affectif, relationnel, rituel, spirituel etc? Même à l'intérieur d'une religion à forte teneur métaphysique, il peut y avoir des croyances, des pratiques, des rites, des formules, métaphysiquement neutres, voire des zones de non-droit métaphysique : " au-delà de cette limite, les concepts métaphysiques ne sont plus valides ». Le problème est le suivant: que signifie, pour une croyance ou un ensemble de croyances religieuses, contenir une conception métaphysique ? Les croyances contiennent-elles des propositions métaphysiques comme le vin contient des sulfites? Sont-elles un ingrédient entrant dans sa fabrication ? OU seulement un produit de son analyse philosophique? La croyance bio serait préservée de tout engrais métaphysique, de tout glyphosate onto-théologique.

A propos de cette implication d'une métaphysique (ou philosophie cosmique) dans la vie de croyance religieuse, on peut d'ailleurs distinguer : a) une implication en troisième personne : tel fait religieux, telle pratique, telle affirmation, semble impliquer aux yeux d'un tiers telle ou telle conception du monde physique, d'un autre monde, etc. et : b) une implication en première personne : l'adhésion explicite de l'adepte, qui vit son engagement religieux comme impliquant un certain nombre de croyances (au moins dispositionnellement, tant il demeure vrai que « l'action par laquelle on croit une chose et celle par laquelle on connaît qu'on la croit étant différentes, elles sont souvent l'une sans l'autre »)

Une même croyance peut donc faire l'objet d'approches divergentes, l'une retiendra des représentations symboliques, l'autre une relation au sacré. L'une énumérera des propositions décrivant la conception du monde véhiculée par cette religion. L'autre privilégiera le récit d'expériences privées, plus ou moins communicables.

Il faut sans doute renoncer à statuer pour "les croyances religieuses en général ».

3. Le statut inférentiel des croyances: croyance intuitive vs croyance réfléchie, et son croisement avec leur degré de transparence (D. Sperber) 
On peut distinguer, avec Dan Sperber ${ }^{4}$, les croyances par leur statut inférentiel : on opposera alors croyance intuitive et croyance réfléchie. Une croyance intuitive est celle à laquelle j'accède directement - je l'ai spontanément, elle n'est pas le résultat d'une enquête ou d'une déduction (je crois que je suis en vie, que j'ai un livre entre les mains, etc.). Une croyance réfléchie, au contraire, résulte de considérations, d'évaluations, de délibérations (je crois que les fantômes n'existent pas, je crois que j'ai bien fait d'acheter ce livre). Par ailleurs, une croyance (spontanée ou déduite) peut être transparente ou opaque. Transparente sera la croyance dont le contenu est clair et distinct. Opaque, celle dont les contours sont indécis, dont on ne sait pas exactement à quoi elle engage, dont on ne saisit pas bien les tenants et els aboutissants. Ce qui est alors en cause, ce n'est plus la manière dont nous accédons à ces croyances (directement ou par réflexion), mais la nature de leur contenu: est-il parfaitement explicite ou contient-il une part d'obscurité ?

On peut alors se demander s'il y a superposition de l'intuition et de l'opacité d'un côté, et de la réflexion et de la transparence de l'autre. Certes on voit mal comment une croyance totalement opaque pourrait être réfléchie, mais l'opacité n'est pas de soi anti-propositionnelle (Sperber parle, à propos de la croyance en une vie après la mort, ou en la Trinité, de croyance semipropositionnelle). Rien n'empêche par ailleurs qu'une croyance intuitive soit transparente.

Je veux simplement suggérer que la croyance religieuse n'est pas nécessairement intuitive (beaucoup de croyances spécifiquement religieuses sont crues sur la base de témoignages de personnes jugées fiables, c'est ce qu'on nomme croyance testimoniale ou, dans certaines typologies, croyance doxastique $^{5}$ - réputationnelle). Et on ne voit pas pourquoi ce type de croyance serait fondamentalement opaque. C'est selon. D'ailleurs, rien n'empêche une croyance intuitive d'être propositionnelle, et à ce titre d'être la base d'une inférence.

\footnotetext{
${ }^{4}$ Dan Sperber, "Les croyances apparemment irrationnelles” dans Le savoir des anthropologues, Paris Hermann 1982.

${ }^{5}$ Cf. Robert Audi, «Belief, Faith and Acceptance », International Journal for Philosophy of Religion February 2008, Volume 63, Issue 1-3, pp 87-102. Audi distingue sept concepts de foi : $1^{\circ}$ ) foi propositionnelle (foi que quelque chose est ainsi) ; $2^{\circ}$ ) foi dans un être (ou une institution); $3^{\circ}$ ) foi « crédentielle » (par laquelle on adhère aux articles d'un credo) $4^{\circ}$ ) foi globale (par laquelle on caractérise une personne de foi) $; 5^{\circ}$ ) foi doxastique, qu'on exerce lorsqu'on croit quelque chose « sur la foi » de quelqu'un ; $6^{\circ}$ ) foi d'acceptation (acceptant faith) qui est en jeu lorsqu'on accueille une personne ou une proposition, et qu'on erxerce sans forcément adhérer à un contenu ou une conviction; $7^{\circ}$ ) foi -allégeance ou foi loyauté, qui est la fidélité, ou la foi gardée envers quelqu'un. Cet article qui décrit admirablement la complexité des concepts de croyance, de foi et d'acceptation est consultable en ligne : https://link.springer.com/article/10.1007/s11153-007-9137-6
} 
Elle le peut, mais ne le sera pas forcément : si je me trouve croire que $p$, et que $p$ implique $q$, il ne s'ensuit pas que je croirai q (j'ignore que q est une condition nécessaire de $\mathrm{p}$ ).

Un autre élément vient perturber la distinction entre croyances opaques et croyances transparentes. Nombre de nos croyances sont latentes: elles existent sous forme de dispositions à croire, qui seront actualisées dans le contexte d'une décision à prendre ou d'un comportement à adopter, ou d'une demande d'éclaircissement. Leur actualisation n'est pas nécessairement synchronique. Leur fausseté ou leur incohérence m'étant révélées, il n'est pas dit que je les révise. Pour la simple raison que ces croyances se présentent rarement dans mon esprit sous la forme d'un système de propositions. Elles se présentent davantage sous la forme d'événements mentaux, d'attitudes récurrentes inégalement réactivées. Je peux donc persister dans un ensemble de croyances incohérentes, parce que je n'entretiens pas toutes ces croyances en même temps et sous le même rapport. Devant un tribunal, je peux être sommé de reconnaître des incohérences entre ce que je crois, ou que je crois croire, et ce que je dis ou fais.

Avant de poursuivre la discussion, je propose de raisonner sur un cas exemplaire cette fois opposant croyance intuitive (en l'occurrence infusée par la grâce) et croyance réfléchie (inférée de connaissances naturelles). C'est l'opposition frontale entre Montaigne et Sebond.

Montaigne retraduit dans les années 1560 la Theologia naturalis seu liber creaturarum (1435) de Raimond Sebon (Ramon Sibiuda ou Sebunde, 13...1436, médecin et professeur d'écriture sainte à l'Université de Toulouse). Il s'agit d'un ouvrage d'apologétique populaire qui n'a rien de scholastique : il est écrit en catalan et ne sera publié en latin que comme posthume. Voici commet Montaigne le recense : «il entreprend, par raisons humaines et naturelles, establir et vérifier contre les atheistes tous les articles de la religion Chrestienne ». En réalité, Sebond essaie de tirer du Livre de nature (l'universel ordre des choses) tout ce qui peut conduire à la connaissance de Dieu et de soi-même. Il faut donc souligner l'exagération de Montaigne : « establir et vérifier tous les articles de la religion ». Tous les articles ? Un programme aussi exorbitant discrédite l'entreprise (qu'on songe seulement aux articles du christianisme qui sont explicitement « seulement révélables » comme la divinité de Jésus).

Par symétrie, Montaigne se range à la revendication d'un accès exclusif par foi et par grâce : « les chrétiens se font tort de vouloir appuyer leur créance par des raisons humaines, qui ne se conçoit que par foy et par une inspiration particulière de la grâce divine » (Essais, II, 12). Comme si tout accès inférentiel était confisqué au profit d'un monopole de l'infusion surnaturelle. Comme si l'examen de motifs de crédibilité, de la recevabilité du témoignage scripturaire ne pouvait en aucun cas entrer en ligne de compte dans l'adoption de telle ou telle croyance professée par les chrétiens. 
L'Apologie très ironique de Raymond Sebon est un peu la matrice d'une opposition qui s'est durcie avec le temps.

Les accusations d'idolâtrie de la raison et de totalitarisme onto-théologique, comme celle de fidéisme ou de délire mystique ont remplacé la courtoisie d'Eyquem.

4. Le procès en idolâtrie conceptuelle

Il peut arriver qu'on décrète l'opacité des croyances religieuses : soit pour les compromettre avec l'infâme superstition, soit au contraire pour les préserver de toute tentative d'analyse conceptuelle et d'examen rationnel. Pour ne pas en rester à de vagues procès d'intention, je commencerai par revisiter un avertissement de Grégoire de Nysse, qui a souvent servi d'exergue comminatoire contre l'invasion des conceptions philosophiques, contre l'hellénisation de la théologie.

L'avertissement de Grégoire de Nysse tiré de son De Vita Moysis est éloquent. Il semble promouvoir un principe fort d'exclusion de tout langage conceptuel, et prôner la destitution de la métaphysique : "Tout concept formé par l'entendement pour tenter d'atteindre et de cerner la nature divine ne parvient qu'à façonner une idole de Dieu, non à le faire connaître ${ }^{6}$. Quelques indications contextuelles sont susceptibles de mitiger l'usage généralement fait de cette déclaration. Le De Vita Moysis est un Traité sur la perfection en matière de vertu, qui médite sur le contraste entre la révélation faite à Moïse et la surdité du peuple : « la théologia [c'est-à-dire ici la parole de Dieu] est une montagne ardue et difficile d'accès, au pied de laquelle la multitude parvient à peine » (Grégoire commente les entretiens de Moïse avec Dieu dans la nuée : «la vision de Dieu consiste à voir qu'il ne peut être vu ». Puis Grégoire explique ce que veut dire : « Moïse entra dans la nuée où

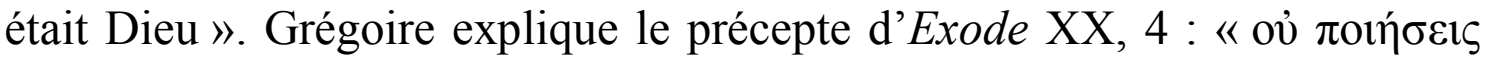

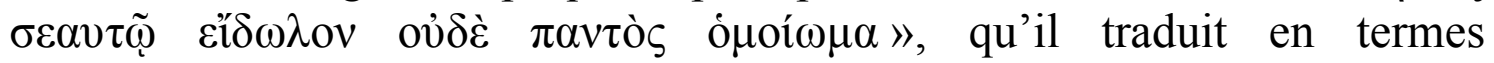
épistémiques : «ne considérer comme semblable à Dieu aucune des choses que l'on connaît ». Grégoire en déduit que la vraie piété consiste d'une part

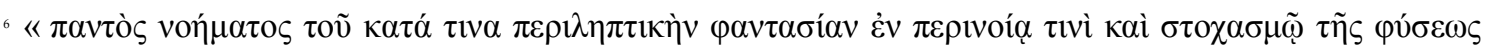

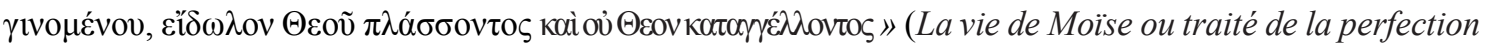
en matière de vertu, introduction et traduction de J. Danielou, Paris 1955 («Sources chrétiennes »), p. 82. L'édition Migne traduit : « omnis enim intellectus, qui phantasia quadam cogitatione, quia si conjecturam divinam naturam apprehendit, simulacrum quidem Dei finxit in mente, Deum autem non intellexit» (Patrologiae cursus completus seu Bibioltheca universalis, integra, uniformis, commoda, oeconomica omnium ss. Patrum, doctorum, scriptorumque ecclesiasticorum, Patrologiae Graecae, apud J.-P. Migne editorem, in via dicta d'AMBOISE, prope portam Lutetiae Parisiorum vulgo d'ENFER nominatam seu PETIT-MONTROUGE, 1863, Tomus XLIV, 377 B-C). L'emploi de «phantasia » renvoie à la théorie stoïcienne de la représentation compréhensive, et la traduction latine est plutôt péjorative, comme d'ailleurs la traduction italienne de ce passage par Antonino Pileri Bruno qui le traduit par « illusion »: « ogni concetto espresso basandosi sull'illusione di averlo acquisito tramite una qualche logica e una qualche riflessio-ne sulla natura è un idolo » (Bruno, Antonino Pileri. "Parola, Immagine e Simbolo Nel Limite Del Tempo e Dell'Eternità." Ho Theològos 35.3 (2017), Print. Studi, p. 373).
} 
en la foi en Dieu, d'autre part en la vie droite : « Ce qu'il faut savoir de Dieu : le connaître, c'est connaître qu'aucune des réalités que l'esprit humain peut connaître n'est Dieu ». On peut donc bien (et même on doit donc bien) savoir quelque chose de Dieu, et déterminer un mode de connaissance très limité, en tout cas pas compatible avec l'exigence de la katalepsis (saisie intuitive complète) ou de la périlèptikè phantasia (représentation complètement délimitée). On devine alors que le principe d'exclusion est plutôt un principe de modération quasi socratique, un rappel qu'on sait qu'on ne sait pas. "Atteindre et cerner » la nature divine, en faire le tour (périnoia) au moyen d'une phantasia katalèptikè, c'est un cahier des charges évidemment trop lourd, qu'il n'est pas très coûteux de dénoncer. Mais on peut fort bien désigner un être ou une réalité au moyen d'une description qui, tout en lui convenant, ne prétend ni la cerner, ni l'enfermer, ni atteindre son essence. Je peux donner un sens et une référence très précises à l'expression « ceux qui m'écoutent» «sans savoir ni ce qu'ils sont, ni s'il y en a ». Toute une littérature apophatique décrit Dieu propositionnellement, en expliquant pourquoi nos façons de parler sont limitées. Pascal lui-même utilise une description de Dieu comme «n'ayant ni parties ni bornes » (ce qui n'est pas donné à tout le monde !) pour affirmer « s'il y a un Dieu, il est infiniment incompréhensible, puisque, n'ayant ni parties ni bornes, il n'a aucun rapport à nous : nous sommes donc incapables de connaître ni ce qu'il est ni s'il est $\gg$.

Il ne faut donc pas exagérer la portée de l'interdit brandi par Grégoire de Nysse. C'est un simple avertissement à ne pas faire de zèle conceptuel alors que l'objet de la croyance déborde manifestement notre capacité d'appréhension. Il la déborde, mais ne la détruit pas. Autrement l'apophatisme tournerait immédiatement à l'aphasie. Diderot saura le rappeler : « Lorsque Dieu, de qui nous tenons la raison, en exige le sacrifice, c'est un faiseur de tours de gibecière qui escamote d'une main ce qu'il a donné $»^{8}$.

\section{Le clivage propositionnalisme/ non-propositionnalisme}

Je propose de revenir pour terminer à l'argument du colloque tel qu'il est exposé dans le programme. Ce programme oppose (pédagogiquement bien

\footnotetext{
${ }^{7}$ Pensées, Lafuma, ${ }^{\circ} 418$. Cf. Cyrille Michon, «Il nous faut bien un concept de Dieu », in Critique n704705, 2006, pp. 92-104. Il est possible d'affirmer que « $x$ est un objet ineffable (unspeakable) dans un langage $l »$. Mais « $x$ pourrait-il être un objet ineffable dans n'importe quel langage $l$ ? ». Selon Bocheński, on peut sans contradiction affirmer : « il existe un objet ineffable dans tout langage », à condition que « tout langage » signifie « tout langage-objet d'une certaine catégorie », et que cette thèse elle-même soit énoncée dans un métalangage correspondant. Un indicible pur (mere unspeakable) est une contradiction. (The Logic of Religion, New York 1965).

${ }^{8}$ Diderot, Additions aux pensées philosophiques ou objections diverses contre les écrits de différents théologiens, III, Paris, GF-Flammarion, 1972, p. 63.
} 
sûr) «réductionnisme méthodologique » et «description phénoménologique ». Selon le réductionnisme (également qualifié de «positiviste»), il est " possible d'isoler un noyau propositionnel fondamental correspondant à une attitude épistémique simple du type $\mathrm{S}$ croit que $\mathrm{p}$, où $\mathrm{p}$ est une proposition constative correctement formée possédant une valeur de vérité ». A contrario, "une description phénoménologique correcte montrerait à quel point la compréhension des attitudes religieuses en termes de croyance propositionnelle est éloignée de la réalité ».

Afin de désamorcer une polémique inutile, je propose la remarque suivante. La question de savoir s'il est possible ou non d'isoler etc... est une question de fait. La réponse à cette question dépend du genre de croyance considérée, et puisqu'on s'intéresse aux attitudes religieuses, à la perception que la personne croyante a de ce qu'elle croit ou de ce en quoi elle croit. Je ne vois pas comment on pourrait a priori statuer sur l'irréductibilité ou non des croyances religieuses à un noyau propositionnel. Il faut demander aux personnes intéressées. Après, libre à chacun de penser que telle attitude est superstitieuse, telle autre victime d'un préjugé onto-théologique, que telle croyance est incohérente, etc. Les philosophes de la religion de tous les pays, continentaux ou non, on une fâcheuse tendance à statuer sur LA foi, LA raison, LA croyance religieuse, comme si la délimitation de ces entités prêtes à la gigantomachie ne posait pas de difficulté. Il est temps de renoncer, au moins provisoirement, à statuer sur LA croyance religieuse, en dehors de tout contexte culturel et confessionnel précis 9 .

On peut toujours soutenir que les attitudes religieuses sousentendent/impliquent des propositions. Au moins les propositions qui les décrivent. Mais il s'agit d'une description en troisième personne. Quant à savoir ce qu'elles impliquent ou non en première personne : seuls les croyants peuvent répondre. Mais que dire d'une personne croyante qui soutiendrait mordicus que ses croyances ne sont pas propositionnellles ? Certes, si on écarte toute description en termes de croyances propositionnelles, on ne sait plus trop ce qu'on va attribuer aux personnes croyantes. L'énoncé par lequel une personne croyante récuse la caractère propositionnel de sa foi est, qu'on le veuille ou non, une proposition. Mais cette proposition ne décrit pas la croyance en elle-même : elle stipule son mode d'emploi. Il est assez probable que l'attitude religieuse soit une combinaison de composantes propositionnelles, émotives, fiduciaires, comportementales (individuelles et sociales), selon un dosage et un déploiement diachronique propre à chaque individu ou à chaque

\footnotetext{
${ }^{9}$ L'attribution de croyance est très conjecturale. Une hindouiste récite un verset de la Baghavad-Gita: que croit-elle exactement? A la vérité littérale de l'histoire du prince Arjuna ? À l'inflexibilité de Brâma ou de Krishna? Au bénéfice d'une récitation auquel elle n'a pas forcément à chercher un sens ?
} 
communauté, sans qu'on puisse les traiter a priori comme des composantes indépendantes.

Telle croyance peut se trouver fragilisée par tel fait, tel accident, telle rencontre. Elle pourra néanmoins, dans certains cas, continuée d'être acceptée. Et le degré d'acceptation n'est pas proportionnel au degré de justification par des raisons. On peut vivre avec une croyance sans que celleci soit étayée rationnellement. Il est plus difficile de vivre avec une croyance rationnellement compromise voire éliminée.

La philosophie analytique de la religion n'est pas spécialisée dans la réduction l'attitude religieuse à un système de propositions soumises à des normes rationnelles. Il y a même un puissant mouvement anti-réductionniste en philosophie analytique. En voici quelques échantillons.

Pour Dewi Zepaniah Phillips, la philosophie de la religion n'a rien à voir avec la question de l'existence de Dieu. Inutile de rechercher une base rationnelle pour les jeux de langage du discours religieux, les formes de vie suffisent : « si le philosophe entend donner une explication de la religion, il n'a qu'à porter son attention sur ce que les croyants font et disent ${ }^{10}$. Phillips conteste l'importation de normes conceptuelles philosophiques « pour tester la validité d'énoncés religieux » Présupposant que ce qui est religieux est non-philosophique , Phillips avoue la difficulté à «rendre compte conceptuelllement d'une activité religieuse familière ${ }^{11}$. " Demander si Dieu existe, écrit-il ailleurs, ce n'est pas poser une question théorique. Si cela peut avoir la moindre signification, c'est s'interroger sur la louange et la prière, c'est se demander s'il y a quelque chose dans tout cela. C'est pourquoi la philosophie ne peut pas répondre à la question « Dieu existe-t-il ? » par l'affirmative ou la négative. "Dieu existe», bien qu'en apparence une déclaration sur le mode indicatif, est une expression de foi. $\rangle^{12}$

Phillips illustre bien la conception non-propositionnaliste des énoncés religieux, si présente dans la philosophie analytique contemporaine de la religion. "Selon la conception non-propositionaliste, le contenu de la révélation n'est pas un corps de vérités concernant Dieu, mais l'intervention de Dieu dans la sphère de l'expérience humaine, au moyen de son action dans l'histoire $\rangle^{13}$. On peut rétorquer qu'une intervention peut toujours être décrite au moyen de propositions, et que donc l'événement, l'expérience, la

${ }^{10}$ D.Z. Phillips, The Concept of prayer, New York, Schocken Books 1966, p. 1.

${ }^{11}$ Ibid., pp. 3, 6-7.

12 D.Z. Phillips, Religion without Explanation, Basil Blackwell 1976, p. 181. C'est un philosophe d'inspiration analytique qui défend la thèse de l'incommensurabilité : les énoncés religieux sont différents des énoncés scientifiques ou métaphysiques, nous ne pouvons pas évaluer les affirmations religieuses avec les normes de la métaphysique (sans parler de celles des sciences !).

${ }^{13}$ John H. Hick, Philosophy of Religion (Fourth Edition), Englewood Cliffs, New Jersey, 1990, p. 64. Voir par exemple N. Wolterstorff (Norton lectures 2000) : "Believers, Theologians and Philosophers : Why Faith has No Need for Philosophy's Grounding" ; "Reason within the bounds of religion"; A. Kenny The Unknown God (2004), ch I: «Ineffable Godhead »; R. Le Poidevin, Religious Fictionalism (2019). 
trajectoire historique ne sont pas condamnées à échapper à la traque propositionaliste! Mais étant donné la variété des «énoncés 》 religieux, je ne vois pas comment on pourrait trancher a priori !

Côté « continental », le congé donné à Dieu est parfois motivé par une prétendue incompatibilité entre la conceptualité philosophique et la pratique religieuse : «Ce Dieu, l'homme ne peut ni le prier, ni lui sacrifier, il ne peut, devant la causa sui, ni tomber à genoux plein de crainte, ni jouer des instruments, chanter et danser ${ }^{14}$. Mais là encore, nous sommes devant une question empirique. Oui ou non, des individus prient-ils, sacrifient-ils, chantent-ils et dansent-ils devant une entité que d'autres désigneront comme cause de soi ? C'est une question de fait, que le philosophe serait bien imprudent de trancher par la négative depuis sa retraite.

Le clivage propositionalistes et anti-propositionalistes ne passe pas entre philosophie continentale et philosophie analytique. Le différend ne porte pas tant sur l'attitude religieuse que sur la manière de faire de la philosophie : en testant des arguments, des raisons d'affirmer ou de nier ou en proposant une vision et en développant une interprétation. Donnons un aperçu de cette opposition de styles. D'un côté une déclaration de Paul Ricoeur : " Dieu doit demeurer l' «absent» de tout discours philosophique $»^{15}$. De l'autre une conclusion d'Anthony Kenny: "Si mon argumentation est correcte, il ne peut exister d'être atemporel, immuable, omniscient, tout puissant et parfaitement bon ». D'un côté on interdit l'usage d'un nom par une proclamation surplombante, étayée par de riches considérations herméneutiques. De l'autre côté, on utilise une description dont on affirme qu'elle n'a pas de référence, argumentation à l'appui. Cette divergence de méthode est bien plus décisive que la position propositionnaliste ou nonpropositionnaliste, comme on a pu en juger sur pièce avec philosophes analytiques (Phillips, Kenny, Le Poidevin). Dans le cadre d'une rencontre entre philosophes de tradition analytique et philosophes d'inspiration herméneutique-phénoménologique, il me semble important de souligner que ce sont moins les thèses que les modes d'exposition et de justification qui les opposent en philosophie de la religion. Sur la question du statut de la croyance religieuse, les uns comme les autres sont sans doute appelés à des enquêtes plus locales, moins spectaculaires que des déclarations sur la foi et la raison, la métaphysique et la croyance. Une cure de nominalisme en quelque sorte.

\footnotetext{
${ }^{14}$ Heidegger, « La constitution onto-théologique de la métaphysique », Identität und Differenz, GA p. 77, trad. dans «Identité et Différence », Questions I, Gallimard, p. 306. Il serait pertinent de rediscuter le diagnostic d'une métaphysique fatalement onto-théologique. Cf. P. Clavier, « L'épuisement de la création : le coup de grâce heideggerien ", Revue Philosophique de Louvain 112(4), 727-749 (nov. 2014).

${ }^{15}$ Paul Ricoeur, La critique de la conviction. Entretien avec François Azouvi et Marc de Launay, Paris, Calmann-Levy, 1996. On peut songer également à Heidegger : « La philosophie elle-même en tant que telle est athée, lorsqu'elle se comprend de manière radicale » (Phaenomenologische Interpretationen zu Aristoteles, Frankfurt, 1985, p. 199). Aucun philosophe théiste n'aurait donc compris de manière radicale la philosophie elle-même en tant que telle ! Étonnant, non?
} 
